\title{
Research on the Teaching Skill of Computer Hardware Technology
}

\author{
Yubian Wang \\ Department of Architecture \\ Belarusian State University of Transport, Belarus. \\ e-mail: wangyubian@qq.com
}

\begin{abstract}
With the development of information technology, the process of accelerating globalization network, computer hardware technology has been an unprecedented development, its application extends to various industries and fields. This paper mainly discuss about the Teaching skill and method of computer technology from the perspective of hardware replacement, analyzes the present situation and existing problems of the current computer technology development, and elaborates the development trend of the future computer in detail.
\end{abstract}

\section{Keywords-Computer Technology; Hardware Development}

\section{A BRIEF INTRODUCTION OF COMPUTER PRODUCTION}

Although the development process of the computer is not long, computer update will have a profound impact on human society. Computer is a pre-stored in accordance with the procedures, automatic, high-speed large-scale numerical calculation and a variety of information processing of modern intelligent electronic devices. Computer can do numerical operations, logical operations, and store data.

\section{A. computer generated}

From the broad perspective, the earliest concept of the computer is a mechanical differential. By the beginning of the 20th century, mathematician Alan Mathiessen Turing proposed a Turing machine model, which provided a computational model for modern computers. The "ideal computer" designed by Turing has also become the originator and prototype of modern computers. In fact, Atanasoff-Berry Computer (ABC) is the world's first electronic computer, appeared in Iowa State University in 1937.This equipment is not programmable, only designed to solve Linear equations, and in 1942 successfully tested.

\author{
Zhichao Lian \\ School of Computer Science and Engineering \\ Xi'an Technological University, Xi'an, 710021, China \\ e-mail: $965941167 @ q q . c o m$
}

Then the world-famous ENIAC was born in the United States on February 14, 1946. ENIAC uses von Neumann structure, can be programmed in advance to automatically perform arithmetic operations, logic operations and storage data functions. The emergence of ENIAC proclaimed the beginning of a new era, marking the advent of the computer information age.

\section{B. Computer development history and status}

Throughout the history of computer development, the computer has brought great changes to people's life. Although the development process is not long, it is extremely rapid and has experienced five important stages of development, and also in the high-speed forward development.

1) Tube computer (1946-1958). This stage of the computer in the hardware is the main feature of the use of electronic components as a basic device, with a light pipe or mercury delay circuit for the main memory.

2) Transistor Computers (1958-1964). Transistor instead of tube as the basic device of the computer, the transistor computer using the core memory as the main memory, drum and disk began to be used as external memory, the overall performance than the tube computer has been greatly improved, and appeared closer to humans Natural language programming language.

3) Small scale integrated circuit computer (1964-1970). The development of semiconductor technology has successfully created integrated circuit, while promoting the rapid development of computer hardware technology. Small and medium-sized integrated circuits become the main components of the computer, the main memory is also gradually transition to the semiconductor memory, greatly reducing the computer computing power consumption, and further improve the reliability of the computer. Disk has become an indispensable auxiliary memory, the computer has entered the product standardization, modular, serial development period, the use efficiency improved 
significantly.

4) Large scale integrated circuit computers (1970 so far). With the successful production of large scale integrated circuits and used in the computer hardware production process, the computer further reduce the size and improve the birth of microprocessors and microcomputers. The computer has a higher flexibility and efficiency, and the emergence of wearable computers, handheld computers and other mobile, devices greatly promoted the development of electronic computers.

5) Artificial Intelligence Computer. Combined with the characteristics of neural networks, artificial intelligence computer can achieve distributed associative memory, and to a certain extent, simulation of human and animal learning function. It is a kind of computer with knowledge, learning and reasoning. It has the ability to understand natural language, voice, text, image and speech, machine can talk directly in natural language, it can take advantage of existing and continuous learning knowledge, thinking, association, reasoning, and come to the conclusion about solve complex problems, with the collection, memory, retrieval of knowledge about the ability.

\section{HARDWARE REPLACEMENT PROCESS}

According to the von Neumann system architecture, modern computer hardware system mainly consists of CPU, memory, I/O equipment and so on. The continuous development of computer hardware system, promote the continuous progress of computer.

\section{A. CPU status and development}

Central Processing Unit (CPU) is a very large scale integrated circuit, is a computer computing core and control the core. Its function is mainly to explain the computer instructions and the processing of computer software in the data.

CPU is the core of the computer components, is generally considered to be an important indicator of the progress of the entire computer hardware technology. According to the word length and function of the microprocessor, its development is divided into the following stages:

The first stage (1971-1973) is a 4-bit and 8-bit microprocessor era, the typical product is Intel4004 and Intel8008 microprocessor, low integration, system structure and instruction system is relatively simple, mainly for simple control occasion.
The second stage (1974-1977) is the 8-bit microprocessor era, its typical product is Intel8080 / 8085, CPU integration and computing speed has improved, with a typical computer architecture and Interrupt, DMA and other control functions, the operating system began to appear.

The third stage (1978-1984) is the 16-bit microprocessor era, its typical product is Intel's 8086 / 8088, integration and computing speed greatly improved, X86 command system more perfect, such as ,Multi-level Interrupt, multiple addressing modes, segment storage mechanism, hardware multiplication and division components, configuration of the software system. By this CPU system, the personal computer has rapid development.

The fourth stage (1985-1992) is the 32-bit microprocessor era. Typical products are Intel's 80386 / 80486, integrated up to 1 million transistors / slices, with 32-bit address lines and 32-bit data bus.

The fifth stage (1993-2005) is the era of the Pentium series of microprocessors, the typical product is Intel's Pentium series chips, the series of CPU internal use of superscalar instruction pipeline structure, has a separate instruction and Data cache, and has a multimedia processing capabilities.

Since 2005, there have been Core Duo series of microprocessors, its typical products Intel's Core i3, Core i5, Core $\mathrm{i} 7$ and Core i9, the CPU has more features to adapt to the rapid development of computer networks and mobile Internet. There are more requirements for the CPU: higher power consumption, higher performance; built-in high-performance GPU; video encoding, graphics performance stronger; Turbo Boost 2.0, more intelligent, more efficient; the introduction of a new ring architecture, bringing higher bandwidth and lower latency ; New AVX, AES instruction set to enhance the floating-point operation .

\section{B. Memory development and current situation}

The development of the CPU drive the other parts of the system constantly upgrading, memory is one of the important components of the computer system. Early use of the "memory chip" was solidified on the motherboard, can not be replaced and installed. Then the emergence of 16-bit microprocessor make the memory performance and capacity 
have a higher demand. The development of the memory module mainly through the following stages:

1) Early memory - SIMM. In the 80286 just launched when the memory using SIMM (Single In-line Memory Modules, unilateral contact memory module) interface, specifications for the 30pin, 256kb.With the CPU into the 32 bit era, 386 and 486 platform using the 72pin SIMM, to solve the problem of low memory bandwidth, but with the upgrade of motherboard and CPU ,SIMM memory has been unable to meet the requirements.

2) Improved memory - EDO. EDO DRAM (Extended Date Out RAM external expansion data mode memory) memory, which is between 1991 and 1995, the prevalence of memory, this memory is mainly used for the ancient Pentium and 486 models, EDO single maximum capacity of only 64M, soon replaced by SDRAM memory.

3) Classic memory - SDRAM full name is synchronous dynamic random access memory, this RAM can make all the input and output signals to keep pace with the system clock. As SDRAM bandwidth of 64Bit, just corresponding to the CPU 64bit data bus width, so it only needs a memory can work, data transfer speed than EDO memory at least $25 \%$ faster.

4) Qualitative leap - DDR series. DDR can be said to be an upgraded version of SDRAM, "double rate SDRAM" , DDR transfers data once on the rising or falling edge of the clock signal means. With the continuous improvement of CPU performance, just rely on high-frequency bandwidth to enhance the DDR has been unable to meet the requirements, therefore DDR2, DDR3 and even DDR4 came into being.

\section{Development and Current Situation of Hard Disk}

Relative to the CPU and memory, hard disk technology development is relatively slow. The current hard drive types are solid state drives (SSDs), mechanical hard drives (HDDs), and hybrid hard drives (based on new hard drives from traditional mechanical hard drives). SSD uses flash memory to store, HDD with magnetic discs to store, mixed hard drive is a hard disk and flash memory together into a hard disk.

Hard drive development has been nearly 60 years of history, the size of the hard disk is getting smaller and the capacity is growing, the hard disk speed and interface technology is also advancing with the times. In 1956, the first piece of hard disk was born, about 60 to 70 years, "Winchester" technology turned out, the first piece of Winchester hard drive came into being, 14-inch hard drive dominated, Winchester technology has become a standard hard drive technology architecture.

\section{Other hardware development status}

In addition to CPU, memory and hard disk, I / O peripherals are also an important part of the computer. Computer peripherals include storage peripherals, communications peripherals, I/O control peripherals, audio and video peripherals etc. With the development of computer hardware, peripherals show a variety of product categories and functional diversification, intelligent control and the appearance of miniaturization.

Diversified peripherals can not be separated from key technology advances. The development of the bus has experienced ISA bus, PCI bus, AGP bus and PCI-Express bus standard, for the I / O device provides a high-speed data channel;

Sound card development process is divided into board, integrated and external three types of interfaces, for different requirements to provide different effects of sound support. Graphics interface standard VGA, PCI and PCI-E etc, provides a more and more high-performance graphics card and display quality; the emergence and development of USB technology, for the diversification of peripherals provides unlimited possibilities, more and more new devices connected to the computer through the USB. In short, the rich external equipment greatly expanded the computer's function, become an indispensable part of computer hardware.

\section{THE DEVELOPMENT OF COMPUTER TECHNOLOGY}

Computer technology is a highly integrated discipline, computer technology presents a miniaturization, intelligent features, and gradually toward the intelligent hardware and Internet of Things development. The development of computer technology mainly presents the following characteristics:

1) Intelligent development of computer technology. The computer can simulate some people's intelligent, and closely linked with human thinking logic, with a certain degree of analysis and learning ability, can make some judgments and reasoning.

2) Network. Network is an important trend in computer 
development. The purpose of networking is to shared the resources.

3) Data processing and numerical calculation of high-speed. The high-speed operation makes it possible to calculate and process data, especially large data. In the Internet era of large data processing thanks to the development of computer technology, through computer-related technology, can achieve effective data processing, speed up the operation speed.

4) Process detection and control. The use of computer technology, can effectively control the production process and business management, production automation, full control and security.

5) Social influence. The emergence of computer technology, especially the computer network has greatly changed the modern way of life, human communication has become extremely relaxed, the transmission of knowledge has become extremely fast, mankind also established a new type of social relations.

\section{PROBLEMS AND SOLUTIONS}

Although the rapid development of computer technology, but there are still some of the following problems:

1) The uneven development of hardware and software. Usually, there are many practitioners in software development, the new software technology is endless, the update speed is extremely fast, and the hardware technology because of various restrictions and bottleneck development is relatively slow, formed a restriction relationship, the hardware technology advances for the development of software technology to provide more Good platform also restricts its development.

2) Reduce costs. Throughout the history of computer development, machine performance is getting higher and higher, the cost is lower and lower, reduce the cost on the one hand to promote the hardware technology innovation, on the other hand can improve the penetration rate of computer technology, more people involved in computer technology research and development.

3) Security and privacy issues. Stand-alone time data can be spread through the mobile media, and the advent of the Internet era makes the data security and personal privacy has become a concern. Therefore, R \& D personnel from the technical to solve the problem, the government departments through legal means to limit the occurrence of such problems, while users themselves should pay attention to self-protection on the Internet.

\section{NEW COMPUTER OUTLOOK}

With the development of parallel technology, the computer system can handle multiple instructions and data at the same time, the speed of operation is greatly improved, the degree of intelligence is getting higher and higher, the processing capacity of the computer is similar, and the silicon chip is close to its physical limit, Architecture and technical experience of a qualitative leap. The development of computers will tend to ultra-high speed, ultra-small, parallel processing and intelligent. In the future, the next generation of computer development direction is as follows:

1) Molecular computer. Molecular computer absorbs the information in the form of electric charge on the molecular crystal and organizes it in an effective way, and realizes the operation process through the interaction process of the protein molecule and the surrounding physical and chemical medium, which has the advantages of small volume, low power consumption and operation fast, large storage capacity and so on.

2) Photonics computer. In the photon computer, the photon replaces the electron, the optical interconnection replaces the wire interconnection, the light operation replaces the electric operation, and uses the different wavelength light to represent the different data, may the high complexity, the large amount of data to achieve the task High-speed parallel processing, the operating speed on the basis of the existing exponential growth.

3) Quantum computer. Quantum computer is a kind of physical device which follows the law of quantum mechanics for high-speed mathematical and logical operation, storage and processing of quantum information. Quantum computers use quantum bits for data storage, so the quantum computer has more storage and higher computing speed than an ordinary computer.

4) Nano-computer. Nano-computer is based on nanotechnology developed a new type of computer. The use of nanotechnology can be atomic-level operation, the derived nanotube components instead of silicon chips to become computer components. With the development of 
science and technology, nanotechnology will eventually be out of the laboratory to promote the development of the computer industry.

5) Biological computer. Biological computer with the computer chip, the storage capacity can reach 1 billion times than the ordinary computer. The density of biological computer components is 100 million times higher than that of brain neurons, and the speed of transmission of information is 100 million times faster than that of human brain. A new generation of computer research has not yet made a breakthrough, but with the continuous development of technology, will be achieved in the near future.

\section{CONCLUSION}

The development of computer technology promote the upgrading of computer hardware, the development of computer from the early numerical calculation to data processing, data organization, processing, retrieval, intelligent control and other tasks for human work and life to bring great convenience. With the continuous development of human society, will make a higher demand for production tools, the computer will be toward the network, intelligent direction.

\section{ACKNOWLEDGMENT}

Fund support: Shaanxi Education Department Special Fund Project number: Shaanxi Education Finance [2013] 23

\section{REFERENCES}

[1] Baidu Encyclopedia. Http: //baiku.baidu.com. Development of mobile intelligent terminal operating system [J] .Zhongxing Communication Technology, 2014.

[2] Information and Computer, 2010. [J]. Information and Computer, 2010.

[3] http://web.gxmu.edu.cn/zz/Article/ShowArticle.asp?

[4] Song Suping, Qin Changhai, etc .. Computer assembly and maintenance. Tsinghua University Press .2014.

[5] http://www.win7china.com/html/2073.html

[6] Wang Zhongsheng. Computer assembly and maintenance. Tsinghua University Press .2011.

[7] Hu Jintao. On the application of computer technology and analysis [A] network security technology and application .2015

[8] Xu Zhenxin, Zhang Shuguan. Moore spirit - the transcendence of silicon world [J]. Chinese computer users .2005.

[9] Li Bin The Research on the Development of Computer Hardware and Related Problems[J].Computer CD soft And the application 2013 (14): 302-304.

[10] Chen Jinrun The Research on Computer Hardware Development [J]. The characters of the times , 2008 (6): 166- 167

[11] Li Qing The Talking about it CPU Development history and computer development prospect [J].Computer Knowledge and Technology Surgery, 2014 (17): 61-63.

[12] Wei Tao. to listen to the workshop-level production management system research and implementation Dalian University of Technology.

[13] Youngest three ways to teach you to view the computer's hardware configuration computer with the network.

[14] Tang Bin, Liu Chao case development highlights Beijing: Electronic Industry Press.

[15] LIU Tao Design and Implementation of Data Loss Recovery Software for Hard Disk Beijing University of Posts and Telecommunications.

[16] Research on High - speed Data Access Technology Based on Hard Disk Array. Nanjing University of Science and Technology.

[17] Research and Application of Solid State Hard Disk. University of Electronic Science and Technology of China.

[18] Design and Research of High Speed Image Data Transmission and Storage System - "Journal of National University of Defense Technology" 